\title{
Ubiquitin in the immune system
}

Julia Zinngrebe, Antonella Montinaro, Nieves Peltzer \& Henning Walczak

Centre for Cell Death, Cancer, and Inflammation (CCCI), UCL Cancer Institute, University College London, London, UK

DOI 10.1002/embr.201470030

Correction to: EMBO reports 15: 28-45 DOI 10.1002/embr.201338025

In this article, published in the January 2014 issue of EMBO reports, there definition of IPS in the Glossary was incorrect. The corrected definition is "IFN- $\beta$ promoter stimulator." In the legend to Figure 2, lines 2 and 3 should read " $\varepsilon$-amino group" instead of " $\alpha$-amino group.” We apologize for any inconvenience caused. 